\title{
Editorial to the special issue: Statistical modeling of environmental extremes
}

\author{
Dan Cooley ${ }^{1}$ (D) . Philippe Naveau ${ }^{2}$
}

Accepted: 17 March 2021 / Published online: 9 April 2021

(C) The Author(s), under exclusive licence to Springer Science+Business Media, LLC, part of Springer Nature 2021

Research in environmental statistics has been a fertile area for the application of the statistical theory of extreme values for many decades. In fact, several original developments in the theory were motivated by problems in environmental science. Because environmental extremes can have tremendous human and ecomomic impact, interest in characterizing extreme phenomena remains as high as ever. Recent methodological developments are motivated by applications of extreme value theory to multivariate, temporal, spatial, and spatio-temporal environmental datasets.

In this special issue devoted to the topic of the "Statistical modeling of environmental extremes," the reader can find seven original articles that treat different environmental statistics:

- Earthquakes and after shocks

- Rainfall extremes

- Severe ocean storms

- High pollutants

- Strong winds

- Daily temperatures

- Floods.

This list highlights the rich variety of applications of extreme value theory.

Each topic raises new questions, and various methodological avenues were explored: univariate extremes (Stein), bivariate extremes (Cai et al.), regionalisation of extremes (Saunders et al.), spatial conditional extremes (Shooter et al.), quantile estimation (Beranger at al.), Bayesian spatial extremes (Chen et al.), and penalised quasi-maximum likelihood estimation (Bucher et al.). This range of topics shows that

Philippe Naveau

philippe.naveau@1sce.ipsl.fr

1 Department of Statistics, Colorado State University, Ft. Collins, CO, 80523-1877, USA

2 Laboratoire des Sciences du Climat et l'Environnement (LSCE) CNRS, Orme des Merisiers / Bat. 701 C.E. Saclay, 91191, Gif-sur-Yvette, France 
cross-pollination between applications, models, and inference techniques can bring new directions and in terms of implementation confirms the saying "In theory, there is not a difference between practice and theory, in practice there is." Although the range of applications and methods is wide ranging, this special issue still only represents a small sample of environmental statistics.

The explosion of data sources in earth sciences will certainly increase the capability of estimating extreme events. This explosion also poses a continued challenge to the EVT community to develop and adapt methods for larger datasets which can contain data of many types and from multiple sources. There is also the continued challenge of adapting recent developments in extremal methods (e.g., incorporation of covariates) to environmental applications. As societal demand for projections of risk due to extreme environmental events will likely continue to increase, the EVT community will need to continue to create and study new statistical tools and concepts.

The guest editors

Dan Cooley and Philippe Naveau

Publisher's note Springer Nature remains neutral with regard to jurisdictional claims in published maps and institutional affiliations. 